\title{
An inexpensive, portable, printing event recorder for behavior studies
}

\author{
D. J. BUCKLEY \\ Engineering and Statistical Research Institute, Research Branch, Agriculture Canada, \\ Ottawa, Ontario KIA OC6, Canada \\ B. D. FRAZER \\ Research Branch, Agriculture Canada, Research Station, \\ Vancouver, British Columbia V6T 1X2, Canada \\ and \\ G. St. AMOUR \\ Engineering and Statistical Research Institute, Research Branch, Agriculture Canada, \\ Ottawa, Ontario KIA 0C6, Canada
}

\begin{abstract}
An inexpensive, field-portable event recorder for monitoring insect or other animal behavior is described. The recorder consists of a 16-key event code keyboard and a 99.99-min timer interfaced to a low-cost printing calculator. The event code number and the elapsed time (.01-min resolution) from the start of an observation period are printed whenever an event key is pressed. The recorder is operable over a temperature range of $0^{\circ} \mathrm{C}$ to $50^{\circ} \mathrm{C}$, with a timing error of less than $\pm .05 \%$ of the $99.99-\mathrm{min}$ full-scale timing range. Periods longer than $99.99 \mathrm{~min}$ can be monitored, provided the return to zero of the timer is noted and taken into consideration.
\end{abstract}

A number of directly computer-compatible behavior recorders have been described (Dawkins, 1971; Gass, 1977; Stephenson, Smith, \& Roberta, 1976; White, 1971). However, these recorders require access to realtime computer systems with appropriate interfaces for subsequent data transcription and analysis, and they of ten are more complex and expensive than practical for recording relatively small amounts of data. Also, these recorders do not provide a printed recording in the field for confirmation of the entered data. Commercial portable data entry terminals with built-in timers are available (e.g., Datamyte 900, Electro/General Corporation, Minnetonka, Minnesota). However, these terminals are expensive $(\$ 2,500)$, and they do not provide for printing of the data at the recording site (see Sidowski, 1977, pp. 403-455). The recorder described here provides a low-cost method of producing printed records of behavior codes and time information. It was designed for monitoring applications in which the amount of data gathered is not sufficient to warrant the cost of more sophisticated recorders.

The recorder, designed primarily for monitoring insect behavior in the field or laboratory, consists of a 16-position event or behavior code keyboard and an elapsed timer interfaced to a low-cost printing calculator. Depression of an event key causes the associated code

This work is Contribution I-86 from the Engineering and Statistical Research Institute, Ottawa, Ontario, Canada. number and the elapsed time from the start of an observation period to be printed on the calculator printer. The printed records can be manually transferred to a computer-compatible medium at a later date for subsequent data processing. The instrument (Figure 1) is battery powered and is housed in a rugged fiberglass case to permit operation in the field.

\section{DESCRIPTION}

The recorder circuitry is shown schematically in Figure 2.

The timer circuit consists of a crystal-controlled clock and divider (U1), a second divider (U2), and a four-digit, binary-coded decimal (BCD) counter (U3, U4). The primary clock signal $(\mathrm{f} 0,32.768 \mathrm{kHz})$ is scaled by dividers in U1 to provide two buffered secondary clock signals ( $\mathrm{fl}$ at $2,048 \mathrm{~Hz} ; \mathrm{f} 2$ at $8 \mathrm{~Hz}$ ) for subsequent timing references. The secondary clock signal, $\mathrm{f} 1$, is further divided by $\mathrm{U} 2$ and fed into the four-digit counter to provide a full elapsed time output of $99.99 \mathrm{~min}$, with a resolution of $.01 \mathrm{~min}$. A switch, $\mathrm{S} 1$, is provided to permit resetting of the timer to zero at the start of an observation period.

The keyboard is encoded in octal notation to simplify the interface requirements. The keyboard is interrogated at a rate of $2,048 \mathrm{~Hz}$ (f1) by a keyboard scanner (U16) operating in conjunction with a counter (U15). The keyboard counter (U15) operates as a binary counter 


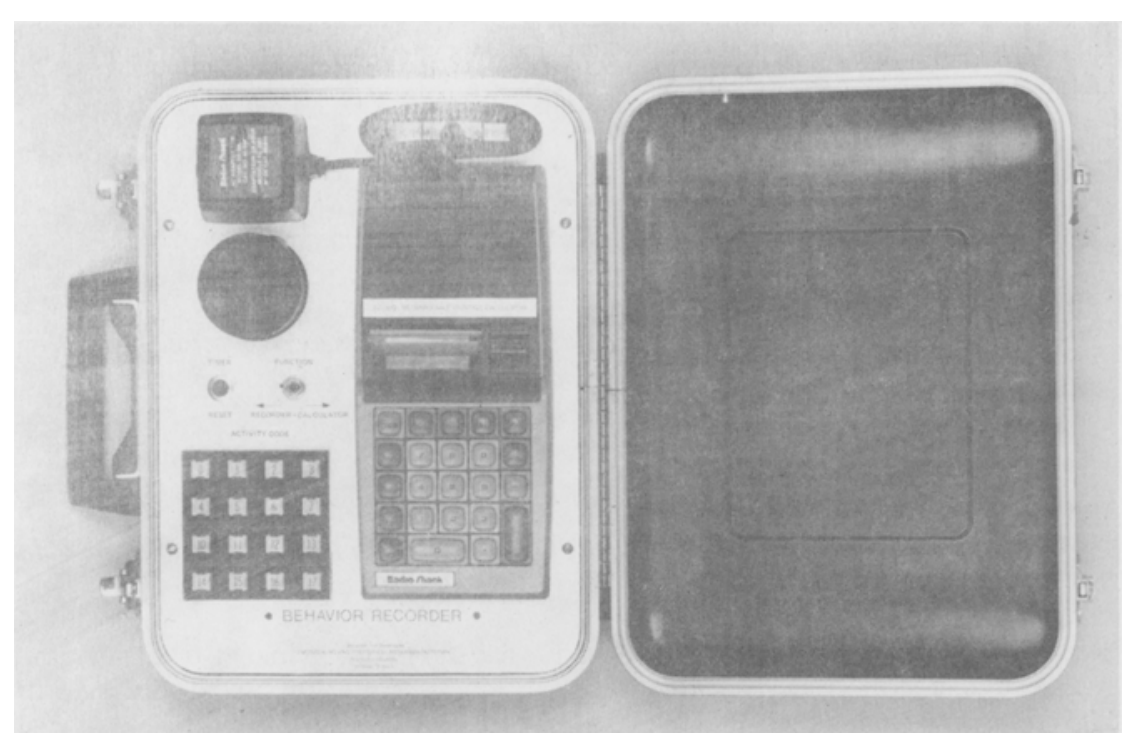

Figure 1. Behavior recorder.

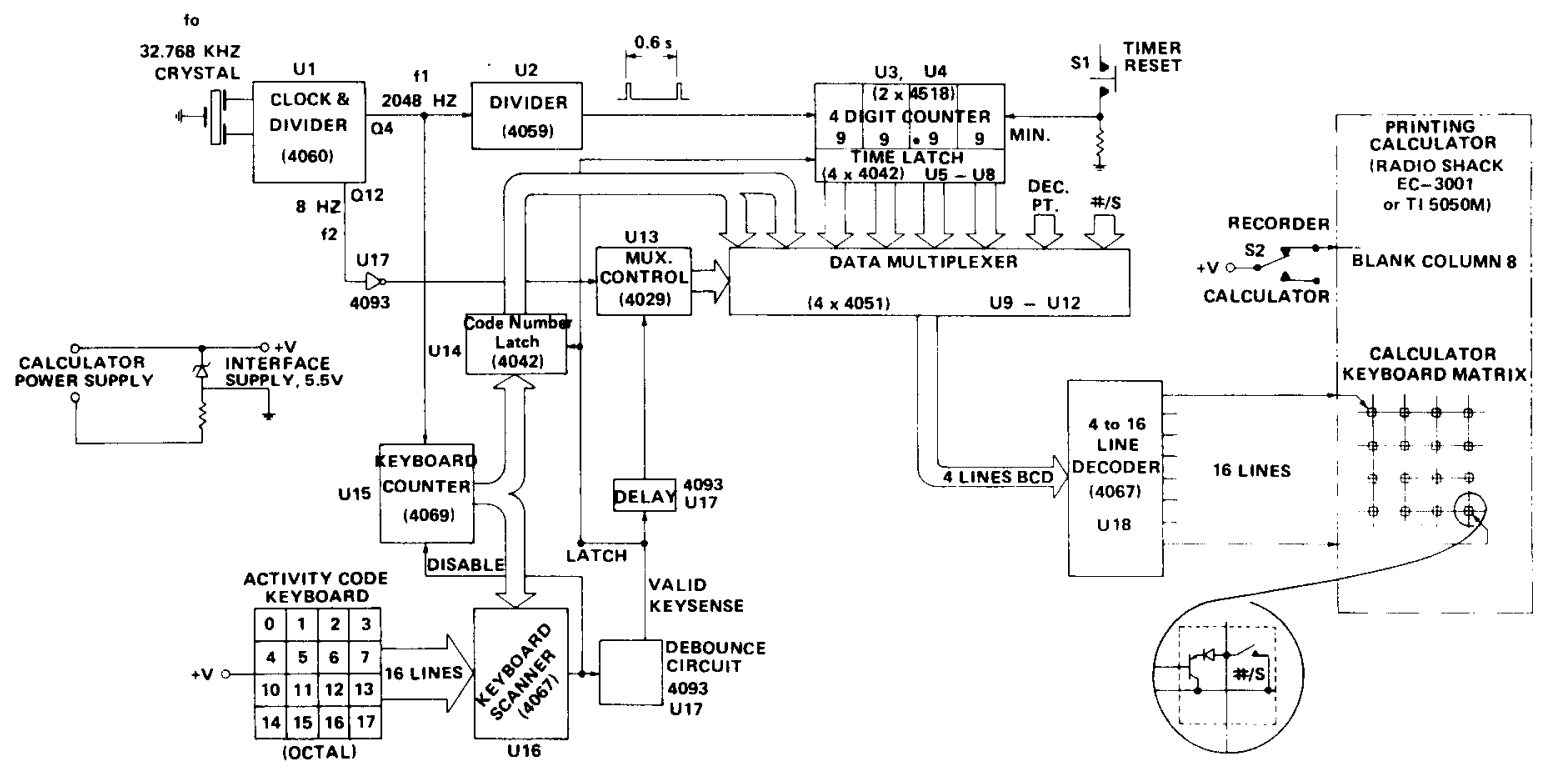

Figure 2. Block diagram of behavior recorder.

driven by the clock signal $\mathrm{fl}$. Whenever an event code key is depressed, the keyboard scanner (U16) outputs a "keysense" pulse when the counter (U15) output corresponds to the key number detected by the keyboard scanner. The keysense pulse, via a switch debounce circuit (U17), disables the counter (U15), latches the octal key number determined by the counter in a code number latch (U14), and latches the current elapsed time in a time-latch circuit (U5-U8). After a short delay (U17) to allow operation of the latch circuits, the latched key number and time data are multiplexed (U9-U12) to the calculator printer through a 4-to-16 line decoder (U18). The data multiplexer (U9-U12) is controlled by a multiplexer control counter (U13) that is enabled by the delayed keysense signal. When enabled, the multiplexer control counter, advanced by the clock signal $f 2$, causes the data multiplexer to sequentially output the latched key number and time digits to the 4-to-16 line decoder. The decoder transfers each BCD data digit to the printer through a transistor 
switch placed across the appropriate number or function in the calculator keyboard matrix. In addition to the six data digits, codes for the decimal point and number sign (\#/S) characters are hard-wired to the data multiplexer. The decimal point character is required to prevent the calculator from blanking significant lagging zeros. The number sign character is used to provide print and paper-advance commands to the calculator.

A thermal printing calculator was selected as the printer because of its low cost (approximately 10\% of that for standard industrial printers at the time of design), low power consumption, proven reliability, and availability. This calculator has been used elsewhere to provide a low-cost printout capability in microcomputer applications (Astmann, 1978; Strelioff, Note 1). This particular printer operates reliably in a rugged environment with a wide temperature range.

A function switch, S2, was incorporated in the instrument to provide a blank space between the time and activity code numbers in the line format of the printed data. The switch also allows the calculator to be used in a calculator mode rather than recorder mode, if desired. The calculator keyboard can also be used to enter fixed data into the recordings (e.g., observation number, date, etc.).

The recorder uses CMOS circuitry throughout for low power consumption. The recorder circuitry external to the calculator is powered, via a Zener regulator, from the calculator's rechargeable battery power supply.

\section{PERFORMANCE AND DISCUSSION}

The recorder was tested over an ambient temperature range from $0^{\circ}$ to $50^{\circ} \mathrm{C}$. The timer error was less than $\pm .05 \%$ of the full-scale timing range of $99.99 \mathrm{~min}$. The calculator printer operated satisfactorily over this temperature range.

The current drain of the circuitry external to the calculator was about $1 \mathrm{~mA}$, which is insignificant compared with the average drain of the calculator when printing frequently. The operating life of the recorder battery power supply, before requiring recharging, depends on the frequency of printing.

Observation periods longer than $99.99 \mathrm{~min}$ can be accommodated provided the operator accounts for the return through zero after each 99.99-min period has elapsed. Variations in the range and resolution of the timer circuitry can be implemented with appropriate circuitry. Similarly, larger capacity keyboards can be implemented with additional interface circuitry to increase the number of available behavior codes. The limitation to such modification would be the number of digits per line available in the printing calculator.

The cost of components for the recorder as described was about $\$ 200$.

\section{REFERENCE NOTE}

1. Strelioff, W. Personal communication, 1978.

\section{REFERENCES}

Astmann, R. H. Interface your computer to a printing calculator. Byte, 1978, 3, 94-99.

Dawkins, R. A. A cheap method of recording behavioral events for direct computer-access. Behavior, 1971, 40, 162-173.

GAss, G. L. A digital encoder for field recording of behavioral, temporal, and spatial information in directly computer-accessible form. Behavior Research Methods \& Instrumentation, 1977, 9, 5-11.

Sidowski, J. B. (Ed.). Behavior research methods \& Instrumentation (Vol. 9). Austin, Tex: Psychonomic Society, 1977.

Stephenson, G. R., Smith, D. P. B., \& Roberta, T. W. The SSR system: An open-format event recording system with computerized transcription. Behavior Research Methods \& Instrumentation, 1976, 8, 259-277.

White, R. E. C. WRATS: A computer compatible system for automatically recording and transcribing behavioral data. Behaviour, 1971, 40, 135-161.

(Received for publication October 9, 1979; accepted October $30,1979$. 